\title{
Large-Scale Preparation of Polymer Nanofibers for Air Filtration by a New Multineedle Electrospinning Device
}

\author{
Yuan Xu, ${ }^{1}$ Xiao Li, ${ }^{1}$ Hong-Fei Xiang, ${ }^{1,2}$ Qian-Qian Zhang, ${ }^{1}$ Xiao-Xiong Wang, ${ }^{1}$ Miao Yu ${ }^{1 D},{ }^{1,3}$ \\ Long-Yun Hao, ${ }^{4}$ and Yun-Ze Long ${ }^{1,4}$ \\ ${ }^{1}$ Collaborative Innovation Center for Nanomaterials \& Devices, College of Physics, Qingdao University, Qingdao 266071, China \\ ${ }^{2}$ Department of Orthopedic Surgery, Affiliated Hospital of Qingdao University, Qingdao 266000, China \\ ${ }^{3}$ Department of Mechanical Engineering, Columbia University, New York, NY 10027, USA \\ ${ }^{4}$ Collaborative Innovation Center for Eco-Textiles of Shandong Province, Qingdao University, Qingdao 266071, China
}

Correspondence should be addressed to Yun-Ze Long; yunze.long@163.com

Received 30 November 2019; Revised 15 February 2020; Accepted 14 March 2020; Published 6 April 2020

Academic Editor: David Cornu

Copyright $\odot 2020$ Yuan Xu et al. This is an open access article distributed under the Creative Commons Attribution License, which permits unrestricted use, distribution, and reproduction in any medium, provided the original work is properly cited.

There are still some challenges for mass-scale production via electrospinning (e-spinning). For example, the cost of industrialized equipment is relatively expensive, and the subsequent maintenance costs are high. The reliability and stability of the production process are also one of the important challenges. The recycling of organic solvents and the volatilization of solvents not only affect the quality of nanofibers, but also causes environmental pollution. In this work, a new multineedle e-spinning device has been proposed for large-scale production of polymer nanofibers. The spinning solution is provided through the outside surface of the needle to avoid needle clogging problem, which is different from the traditional multineedle e-spinning. The successful preparation of thermoplastic polyurethane (TPU) nanofiber membrane with production rate $\sim 50 \mathrm{gh}^{-1}$ proves the feasibility of the device, which also can be used to prepare other functional nanofibers such as polyvinylidene fluoride (PVDF) and polyacrylonitrile (PAN). The prepared TPU nanofiber gauze has been characterized. The average fiber diameter was $145.3 \mathrm{~nm}$. The surface of the sample was found to be uniform, and the water contact angle was $138.9^{\circ}$. The sample had gas permeability of $1500 \mathrm{~mm} \mathrm{~s}^{-1}$, excellent PM2.5 removal efficiency of $99.897 \%$, and optical transparency of $\sim 56 \%$, indicating that the new device has a practical application perspective.

\section{Introduction}

Following the invention of electrospun (e-spun) nanofibers in 1934 by Thomas [1], electrospinning (e-spinning) technology was in a trough for a long time. In recent decades, e-spun nanofibers have been extensively studied by researchers, and their potential applications have also been largely studied [2-7]. Today, e-spinning equipment and technical solutions, as well as e-spun materials, are rapidly turning to commercialization. At the same time, it is not limited to laboratory preparation of nanofibers, but also specialized companies provide laboratory and industrial-scale components and equipment for e-spinning and commercialize their batch-prepared nanofibers. Therefore, researchers are committed to how to solve various technical problems in the mass production process and increase the production rate of nanofibers, which is a key issue for commercial applications [8]. At present, the solution to the above problems is mainly to continuously improve the development of nanofiber batch manufacturing technology through the innovation of multiple devices and improvement of espinning nozzles, so as to provide ideas for the industrial manufacturing of nanofibers.

For the time being, most of the e-spinning devices in lab use a single needle method to produce nanofibers, which makes the production and efficiency of nanofibers very low and cannot meet the requirements of large-scale production. The method of realizing multinozzle and multijet by improving the device can not only greatly improve the yield of nanofibers, but also achieve continuous production and obtain large-area fiber mesh [9-12]. The multinozzle or multijet method e-spinning refers to the purpose of spinning a plurality of nozzles according to a 


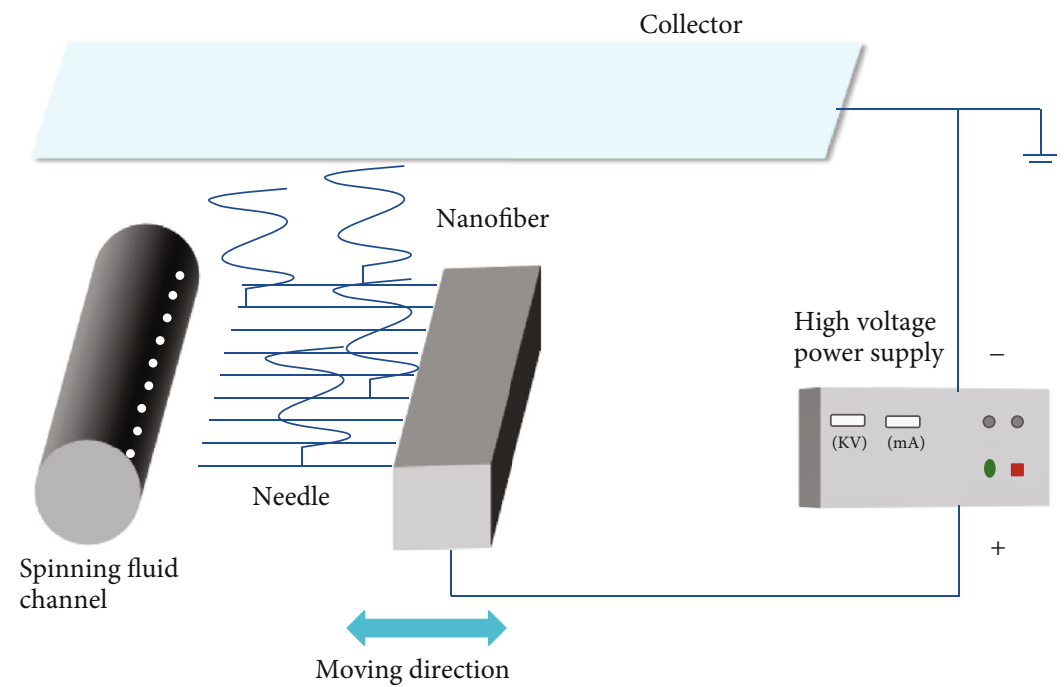

FIGURE 1: Schematic diagram of the multineedle external liquid e-spinning device.

certain regularity, thereby achieving the purpose of batch preparation of nanofibers. Multineedle array spinning is generally performed in a linear or two-dimensional array (e.g., triangular, hexagonal, and circular) [13]. Theron et al. [11] designed a method of e-spinning nanofibers into a $3 \times 3$ array of needles, resulting in a yield that was several hundred times greater than that of a single nozzle. Li et al. [14] designed an e-spinning device that is different from the traditional multineedle linear arrangement. The device uses a roller as the receiving pole, and the linearly arranged needles are mounted on the laterally moving slide table. Then, the polystyrene is obtained by adjusting the number of nozzles of different polymer solutions. The nanofiber membrane is uniformly mixed with the polyamide two-component, and the functionalized fiber is obtained while increasing the yield. Kim and Park [15] studied a similar multineedle up-spinning device, which expanded the number of needles to 38,880, greatly promoting the industrial scale of multineedle e-spinning produce. Liu et al. $[16,17]$ designed a mesh belt multineedle e-spinning device, by controlling the volatilization rate of the polymer solution by installing a temperature control system on the e-spinning device. The production quality of the fiber web is improved, and it is also proved that the device can be simultaneously spun using a plurality of solutions to obtain a composite nanofiber web. Liu et al. [18] proposed a high-efficiency needle-free e-spinning technology based on a circular rotary bead wire for the preparation of polymer nanofibers. This needle-free e-spinning technology is expected to produce nanofibers on a large scale. Important advances in needle-free spinning methods, including spinneret construction, production efficiency, fiber quality, and available industrial-scale equipment and commercial applications, are highlighted in the market [19].

It is worth noting that several spinning devices for mass production of nanofibers in the industry currently have certain disadvantages. For example, conventional e-spinning provides a polymer solution through the inside of the needle, and there are often problems such as needle clogging. For the drum-type electrode spinning, although the spinning yield is improved, the open solution tank in the spinning process has the disadvantage of solvent volatilization, which leads to a gradual increase of the spinning solution concentration and affects the fiber morphology.

In this work, according to the various multineedle espinning devices previously reported, a new multineedle external liquid device was designed. The experiment was carried out with thermoplastic polyurethane (TPU) solution as the e-spinning precursor solution, which proved the feasibility of the device and successfully produced nanofibers. At the same time, the efficiency of preparing the nanofibers is greatly improved, and the nanofiber membrane can be prepared quickly and continuously. The prepared nanofiber membrane was characterized, and the hydrophobicity test proved that the prepared nanofiber had better hydrophobicity. Through the test of translucency of the sample, the anticaries and gas permeability cycle test proved that it has good sustainable use, remarkable PM2.5 removal efficiency (99.897\%), air permeability $\left(1500 \mathrm{~mm} \mathrm{~s}^{-1}\right)$, and optical transparency (56\%) that can be used in commercial scale production of antifog screens.

\section{Experimental Section}

2.1. Experimental Device Composition and Principle. The experimental device is shown in Figure 1. First, the device components include a cylindrical spinning solution channel (with a diameter of $1.0 \mathrm{~cm}$ ), a linear multineedle slide table (the needle diameter is about $1.0 \mathrm{~mm}$, and the needle spacing is about $4.0 \mathrm{~cm}$ ), a high-voltage power supply, a liquid collection tube, and a collector (the needle-collector distance is about $30 \mathrm{~cm}$ ). The positive pole of the high-voltage power supply is connected to the multineedle slide table, and the negative pole receives the collector. The cylindrical channel is filled with polymer solution, the spinning solution channel is fixed, and the multineedle sliding table continuously draws the polymer solution by moving left and right (the moving speed is about $0.2 \sim 0.5 \mathrm{~m} \mathrm{~min}^{-1}$ ), so that the spinning solution 


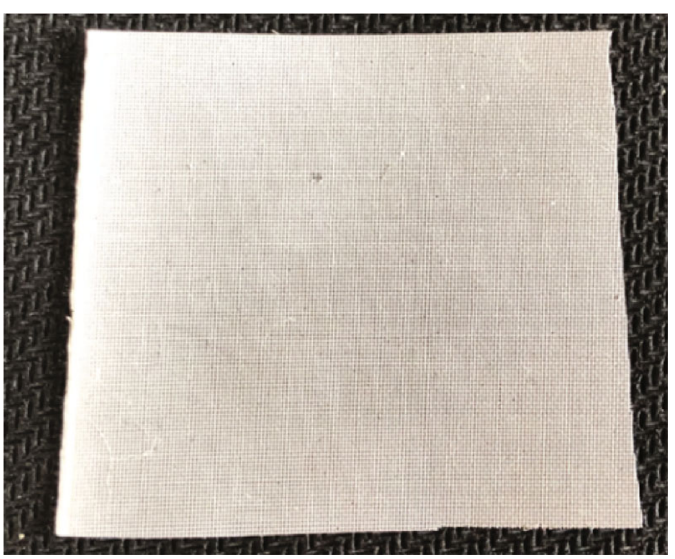

(a)

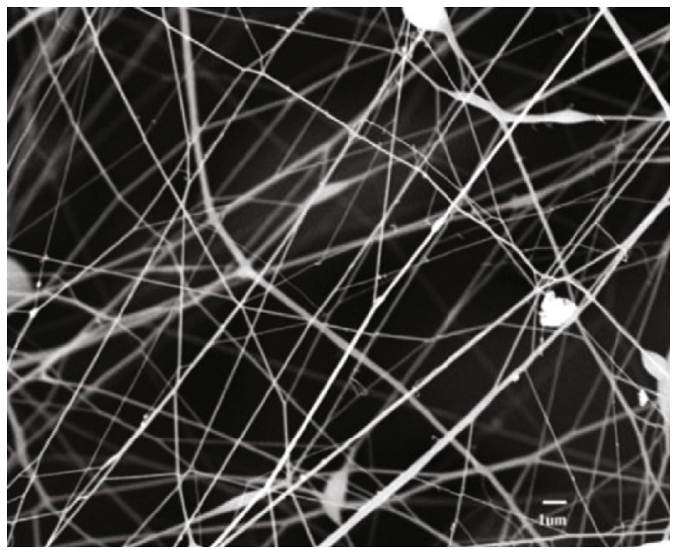

(c)

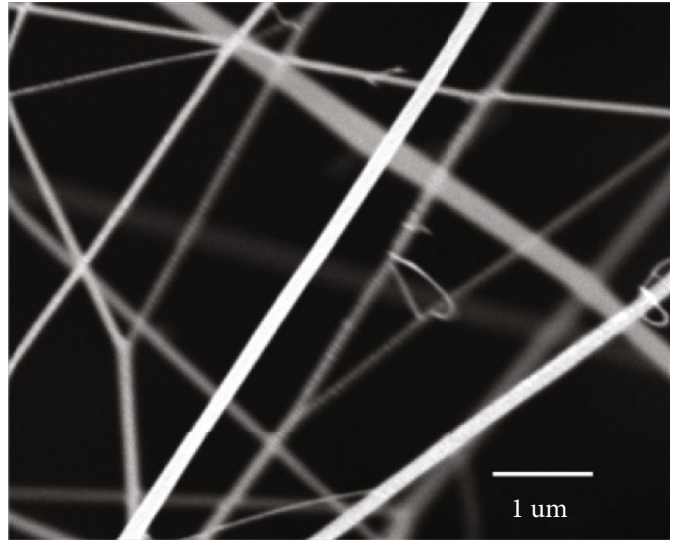

(b)

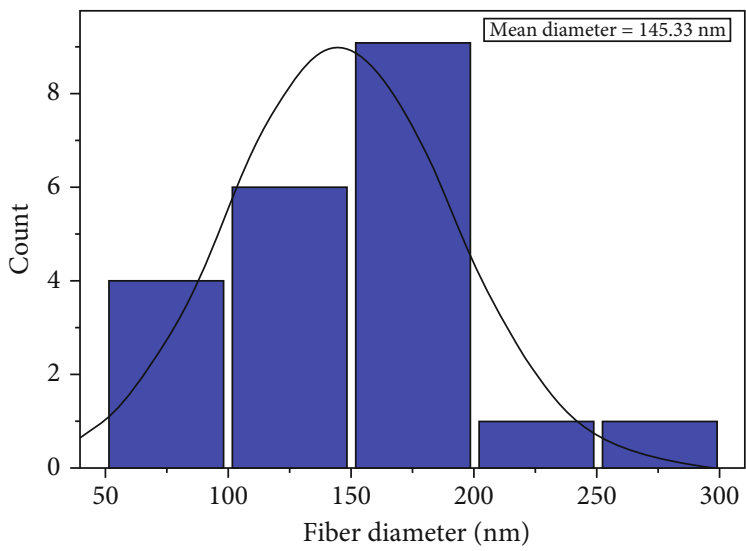

(d)

FIGURE 2: Morphology of e-spun TPU fiber membrane. (a) Optical image. (b, c) SEM pictures of the TPU fibers. (d) Diameter distribution of the fiber.

in the cylindrical channel is externally attached to the surface of the long needle. When a high voltage $(50 \sim 60 \mathrm{kV})$ is applied, the plug fluid forms a jet under the traction of a high-voltage electrostatic field and eventually solidifies into a collector deposited on the fiber. In this process, each long needle surface can form multiple jets at the same time, so the multineedle linear arrangement can effectively improve the preparation yield of nanofibers.

2.2. Experimental Equipment and Spinning Conditions. The morphology of TPU fibers was investigated by scanning electron microscopy (SEM Feiner High Resolution Professional Edition Phenom Pro). The FX3300 laboratory Air-IV automatic filtration performance tester from Shanghai Lippo Co., Ltd. was purchased to evaluate the filtration performance. The air permeability of Shanghai Huifen Electronic Technology Co., Ltd. was tested using AFC-131. The TPU fiber film contact angle was analyzed by an optical contact angle meter. The light transmittance of the Shanghai Aoxiang Scientific Instrument Co., Ltd. UV1901PC ultraviolet spectrophotometer was measured.
TPU was used as the spinning material in the experiment. First, the TPU was dissolved in DMF, the TPU mass fraction was $17 \mathrm{wt} \%$, and the mixture was stirred at $40^{\circ} \mathrm{C}$ until completely dissolved. The applied high voltage is $60 \mathrm{kV}$, and the receiving distance was $30 \mathrm{~cm}$. In the experimental process, a TPU nanofiber gauze was obtained by preparing a TPU fiber using a fine gauze as a receiver.

\section{Results and Discussion}

3.1. Fiber Morphology Characterization. As shown in Figure 2, we successfully prepared TPU nanofibers through a multineedle external liquid device. Figure 2(a) shows an optical picture of the fiber membrane, and it is apparent from the picture that the surface of the fiber membrane is uniform. Figures 2(b) and 2(c) are SEM images of fiber membranes at different magnifications, and Figure 2(d) is a fiber diameter distribution diagram of TPU fibers. From the fiber diameter distribution diagram, it can be seen that the average diameter of the TPU fiber membrane is $145.3 \mathrm{~nm}$, the diameter of the TPU fiber prepared by other methods is finer, and the fiber 


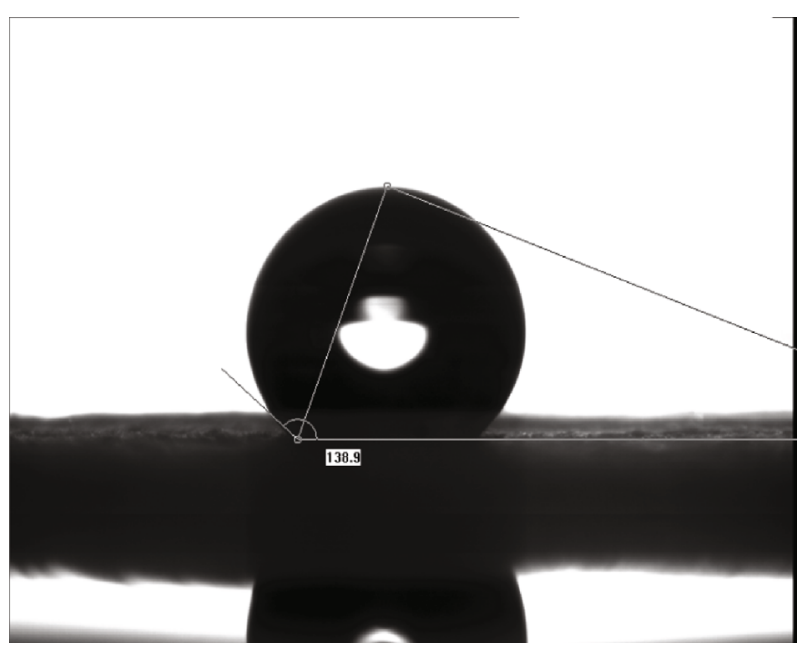

FIgURE 3: The water contact angle of the TPU fiber gauze.

distribution is uniform. The average diameter of the nanofibers of the traditional needle electrospun TPU prepared in our earlier stage is about $1094 \mathrm{~nm}$ [20]. Obviously, the diameter of nanofibers prepared by multineedle electrospinning is smaller.

3.2. TPU Fiber Gauze Hydrophobicity Test. The prepared TPU nanoyarn web was tested for contact angle, and its hydrophobic property was judged. The contact angle of water to the water was $138.9^{\circ}$, which proved that the TPU nanogauze had great hydrophobic properties, as shown in Figure 3. The water contact angle of traditional electrospun TPU nanofibers is $129^{\circ}$ [20]. The excellent hydrophobic properties of TPU nanofiber gauze provide a strong guarantee for rain-proof screens in subsequent real life, and the hydrophobic properties provide the possibility of recycling of TPU nanostencils and extend the cycle and service life. In order to further simulate the resistance of the material to different pollutants in rainy days, we explored the hydrophobic properties of different kinds of dye solutions. We observed the fiber membrane by optically photographing water droplets and different kinds of dye solutions (including rhodamine $\mathrm{B}$, methylene blue, and methylene orange) onto the TPU nanoyarn net. The hydrophobic properties confirmed that the TPU nanofiber gauze has better hydrophobic properties for the abovementioned liquids, as shown in Figure 4.

3.3. Transmittance Test. The transmittance test of the TPU nanofiber gauze was carried out. Figure 5(a) shows an optical picture of the light transmission of a blank gauze, and the light transmission of the blank gauze is $80 \%$. Figure $5(\mathrm{~b})$ is a transparency optical photograph of the TPU nanofiber gauze. The optical fiber screen of the prepared nanofiber gauze was $56 \%$, and the air filter screen with a general transmittance of $50 \%$ or more was able to pass sufficient light. It is proved to have excellent light transmittance. The TPU nanofiber film prepared by tradi- tional electrostatic spinning has a light transmittance of $60 \%$, which is not much different [21].

3.4. Filtration Performance and Permeability Test. Filtration efficiency is one of the important parameters for evaluating the performance of antifog gauze. Therefore, we conducted a filtration efficiency test on the prepared TPU nanofiber gauze. During the test, the temperature was $20^{\circ} \mathrm{C}$, the relative humidity was $40.6 \%$, the air flow rate was set to $2.0 \mathrm{~m}^{3} \mathrm{~h}^{-1}$, and the aerosol particles were used as PM contaminants. Figure 6 shows the filtration efficiency and filtration schematic of the TPU nanofiber gauze. Figure 6(a) shows the filtration efficiency of the sample is tested for 10 times. It can be seen from Figure 6(a) that the filtration effect of the sample is proportional to the particle size, and the larger the particle size, the more difficult it is to pass through the nanofiber membrane. When the particle diameter is $\geq 3.75 \mu \mathrm{m}$, the removal efficiency is $100 \%$, and the pressure drop is only $20 \mathrm{~Pa}$. For PM with smaller particle size, the removal effect is also higher, with a minimum of $96.3 \%$. At the same time, it can be seen that the removal rate of PM2.5 by TPU nanofiber gauze is $99.897 \%$. According to literature reports, the filtration efficiency of PM2.5 for TPU composite nanofiber membranes prepared by traditional electrostatic spinning technology is $99.654 \%$ [21]. Therefore, the nanofiber membrane prepared by the new multineedle electrostatic spinning technology has better filtration performance. Figure 6(b) is a schematic diagram of the effect of TPU nanofiber gauze on PM filtration of different particle sizes.

The recyclability of nanofiber gauze is also an important parameter to measure whether it can be used as an antifog screen for commercial production. Therefore, we performed a filtration efficiency cycle test and a gas permeability cycle test on the PMU nanofiber gauze for PM2.5, as shown in Figure $7(\mathrm{a})$. The graph shows the removal efficiency curve of the sample-filtered PM2.5 for 10 cycles. After 10 cycles of filtration, the filtration efficiency was only reduced by about $0.4 \%$ (from $99.897 \%$ to $99.516 \%$ ). In addition, the gas permeability error bars of the 10 test cycles of the TPU nanofiber gauze are shown in Figure 7(b). Before the first filter gas permeability measurement, the sample was measured to have a gas permeability of $1500 \mathrm{~mm} \mathrm{~s}^{-1}$ without the first filtration. The measurement pressure of the gas permeability test is $200 \mathrm{~Pa}$, and the measurement interview is $20 \mathrm{~cm}^{2}$. After ten cycles of filtration, it can be seen that the change in gas permeability is slow, indicating that the air permeability is still good.

\section{Conclusions}

So far, multinozzle or multijet e-spinning technology is one of the most common methods to increase nanofiber production and promote large-scale production of e-spun fibers. In this work, a new multineedle e-spinning method has been proposed to prepare polymer nanofibers, which greatly improves the fiber production efficiency. The water contact angle of the prepared TPU nanofiber gauze was $138.9^{\circ}$, which proved that the sample had hydrophobicity. Particularly, the TPU nanofiber gauze prepared by this device has excellent recyclability and remarkable PM2.5 removal efficiency 


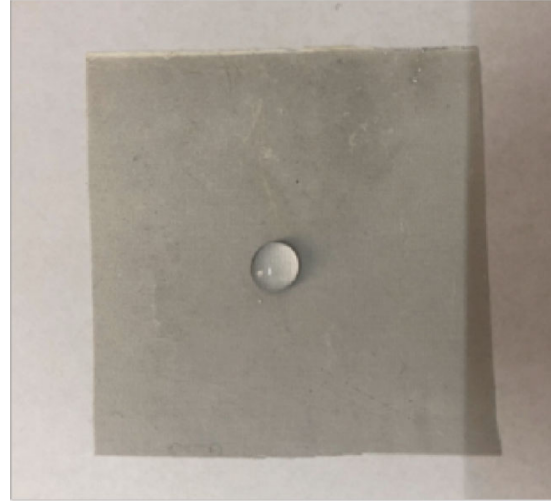

(a)

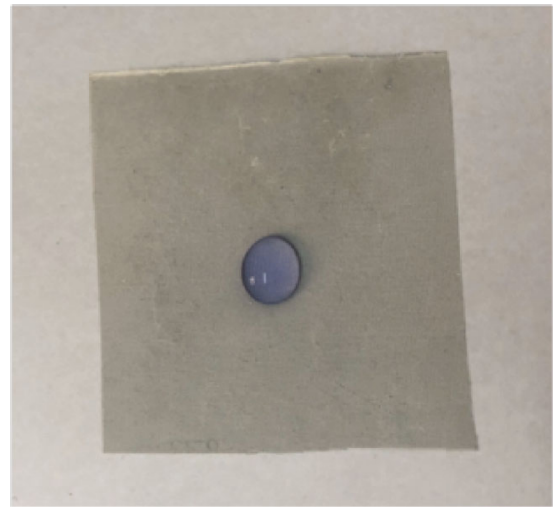

(c)

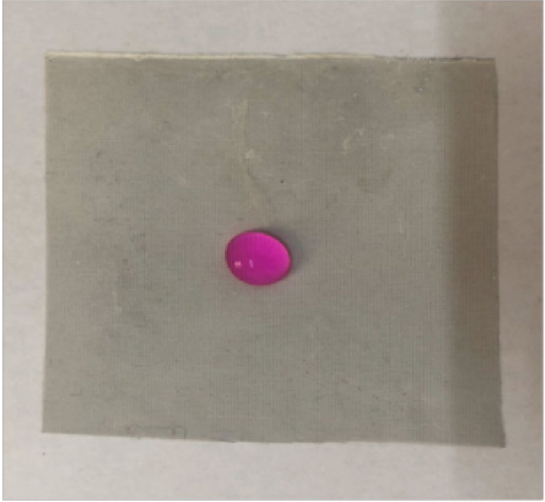

(b)

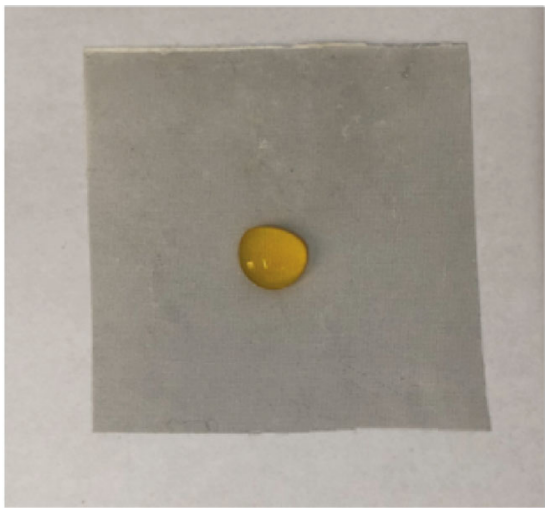

(d)

FIGURE 4: Optical photographs showing the hydrophobic angle characteristics of different types of dyes. (a) Water. (b) Rhodamine B. (c) Methylene blue. (d) Methylene orange.

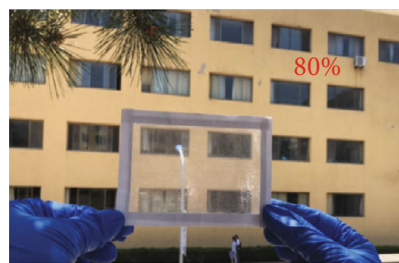

(a)

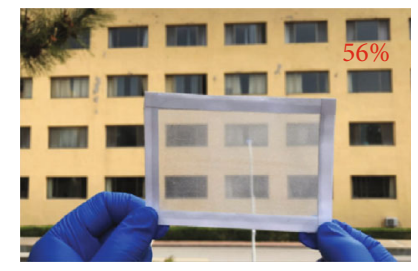

(b)

FIgUre 5: Optical picture of transmittance. (a) Blank gauze. (b) TPU nanofiber gauze.

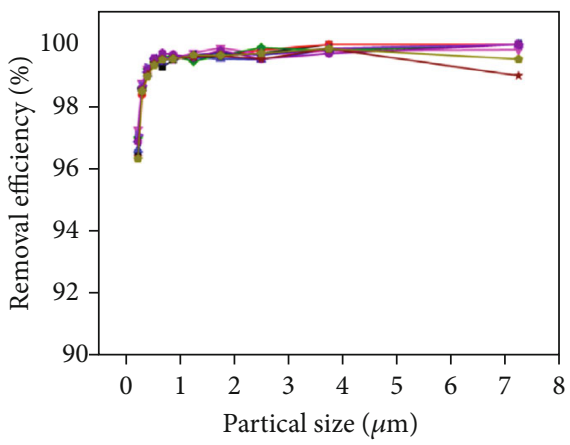

(a)

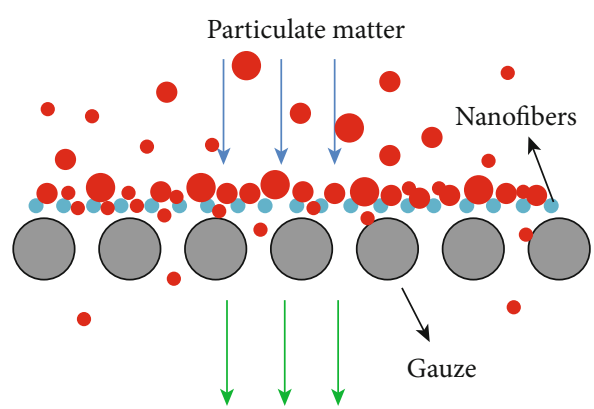

(b)

FIGURE 6: Filtration performance of TPU nanofiber gauze. (a) The different times of filtration efficiency test. (b) Schematic diagram of PM filtration for air. 


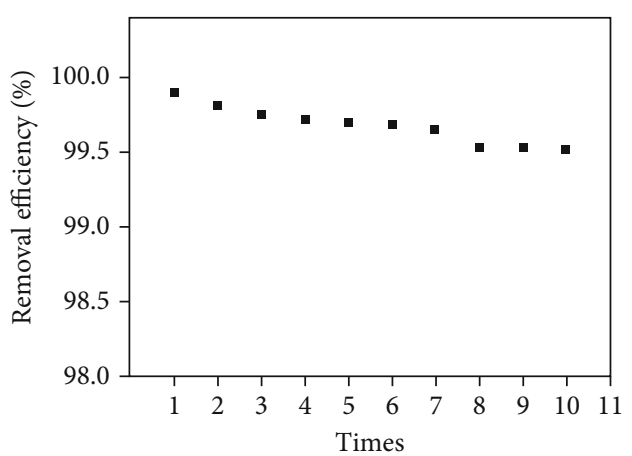

(a)

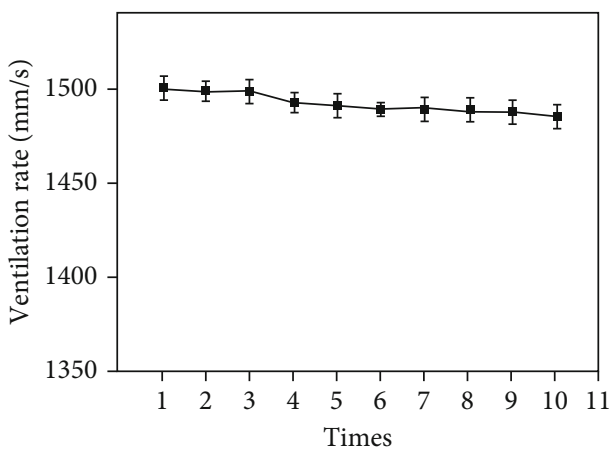

(b)

Figure 7: (a) Cycle diagram of filtration efficiency of the sample. (b) Cycle diagram of ventilation rate of the sample.

(99.897\%), gas permeability (1500 $\left.\mathrm{mm} \mathrm{s}^{-1}\right)$, and optical transparency $(\sim 56 \%)$. The new e-spinning device may be used for large-scale production of polymer nanofibers and antifog screens.

\section{Data Availability}

The data used to support the findings of this study are included within the article.

\section{Conflicts of Interest}

The authors declare that they have no conflicts of interest.

\section{Authors' Contributions}

Yuan $\mathrm{Xu}$ and Xiao Li contributed equally to this work.

\section{Acknowledgments}

This work was supported by the National Natural Science Foundation of China (51973100 and 51673103) and the Postdoctoral Scientific Research Foundation of Qingdao (2017009).

\section{Supplementary Materials}

Figure S1: picture of a new multineedle spinning device for electrospinning process. Table 1: comparison between traditional multineedle electrospinning and new multineedle electrospinning. (Supplementary Materials)

\section{References}

[1] F. Anton, "Process and apparatus for preparing artificial threads," US Patent US50028330A, 1934.

[2] X. X. Wang, W. Z. Song, M. H. You et al., "Bionic singleelectrode electronic skin unit based on piezoelectric nanogenerator," ACS Nano, vol. 12, no. 8, pp. 8588-8596, 2018.

[3] T.-T. Yue, X. Li, X.-X. Wang et al., "Electrospinning of carboxymethyl chitosan/polyoxyethylene oxide nanofibers for fruit fresh-keeping," Nanoscale Research Letters, vol. 13, no. 1, p. 239,2018
[4] B. Zhang, Z.-G. Zhang, X. Yan et al., "Chitosan nanostructures by in situ electrospinning for high-efficiency PM2.5 capture," Nanoscale, vol. 9, no. 12, pp. 4154-4161, 2017.

[5] X. Li, X. X. Wang, T. T. Yue et al., "Waterproof-breathable PTFE nano- and microfiber membrane as high efficiency PM2.5 filter," Polymers, vol. 11, no. 4, p. 590, 2019.

[6] M. H. You, X. X. Wang, X. Yan et al., “A self-powered flexible hybrid piezoelectric-pyroelectric nanogenerator based on non-woven nanofiber membranes," Journal of Materials Chemistry A, vol. 6, no. 8, pp. 3500-3509, 2018.

[7] H. J. Qiu, W. Z. Song, X. X. Wang et al., “A calibration-free self-powered sensor for vital sign monitoring and finger tap communication based on wearable triboelectric nanogenerator," Nano Energy, vol. 58, pp. 536-542, 2019.

[8] D. Fang, B. S. Hsiao, and B. Chu, "Multiple-jet electrospinning of non-woven nanofiber articles," Polymer, vol. 44, no. 2, pp. 59-60, 2003.

[9] O. O. Dosunmu, G. G. Chase, W. Kataphinan, and D. H. Reneker, "Electrospinning of polymer nanofibres from multiple jets on a porous tubular surface," Nanotechnology, vol. 17, no. 4, pp. 1123-1127, 2006.

[10] C. Burger, B. S. Hsiao, and B. Chu, "Nanofibrous materials and their applications," Annual Review of Materials Research, vol. 36, no. 1, pp. 333-368, 2006.

[11] S. A. Theron, A. L. Yarin, E. Zussman, and E. Kroll, "Multiple jets in electrospinning: experiment and modeling," Polymer, vol. 46, no. 9, pp. 2889-2899, 2005.

[12] B. Ding, E. Kimura, T. Sato, S. Fujita, and S. Shiratori, "Fabrication of blend biodegradable nanofibrous nonwoven mats via multi-jet electrospinning," Polymer, vol. 45, no. 6, pp. 18951902, 2004.

[13] W. Tomaszewski and M. Szadkowski, "Investigation of electrospinning with the use of a multi-jet electrospinning head," Fibres and Textiles in Eastern Europe, vol. 13, no. 4, p. 22, 2005.

[14] X. Li, B. Ding, J. Lin, J. Yu, and G. Sun, "Enhanced mechanical properties of superhydrophobic microfibrous polystyrene mats via polyamide 6 nanofibers," The Journal of Physical Chemistry C, vol. 113, no. 47, pp. 20452-20457, 2009.

[15] H.-Y. Kim and J.-C. Park, "Conjugate electrospinning devices, conjugate nonwoven and filament comprising nanofibers prepared by using the same," US Patent US20080102145, 2008.

[16] C.-K. Liu, K. Lai, R.-J. Sun, and M.-Y. Chen, "Investigation on process of multi-needle electrospinning," Journal of Textile Research, vol. 33, no. 8, pp. 7-10, 2012. 
[17] C.-K. Liu, K. Lai, R.-J. Sun, and M.-Y. Chen, "Preparation of aligned nanofiber assembly through electrospinning and its mechanism," Polymer Materials Science \& Engineering, vol. 28, no. 6, pp. 117-120, 2012.

[18] S. L. Liu, Y. Y. Huang, H. D. Zhang, B. Sun, J. C. Zhang, and Y. Z. Long, "Needleless electrospinning for large scale production of ultrathin polymer fibres," Materials Research Innovations, vol. 18, Supplement 4, pp. 833-837, 2014.

[19] M. Yu, R. H. Dong, X. Yan et al., "Recent advances in needleless electrospinning of ultrathin fibers: from academia to industrial production," Macromolecular Materials and Engineering, vol. 302, no. 7, article 1700002, 2017.

[20] M. N. Liu, X. Yan, M. H. You et al., "Reversible photochromic nanofibrous membranes with excellent water/windproof and breathable performance," Journal of Applied Polymer Science, vol. 135, no. 23, article 46342, 2018.

[21] W. Liang, Y. Xu, X. Li et al., "Transparent polyurethane nanofiber air filter for high-efficiency PM2. 5 capture," Nanoscale Research Letters, vol. 14, no. 1, p. 361, 2019. 